\title{
Uses of Single Dose Dependent and Relative Potency Assays for Evaluation of Inactivated Fowl Cholera Vaccine
}

\author{
Selim S. Salama*, Fatma Mohammed Gadallah, Fatma El-Zahraa Gamal Abo-Elkhir, \\ Afaf Ahmed Khedr and Mahmoud A. Ali \\ Central Laboratory for Evaluation of Veterinary Biologics, Abbasia, Cairo, Egypt \\ *Corresponding author's Email: selimsalama2000@yahoo.com; ORCID: 0000-0001-8797-2029
}

Received: 05 Jul. 2019

Accepted: 12 Aug. 2019

\begin{abstract}
Fowl cholera is a septicemic respiratory complex caused by Pasteurella multocida, widely distributed in poultry and other avian species and of major economic importance. A total of 37 different inactivated Pasteurella multocida vaccines from different sources either locally prepared or imported from different sources were comparatively tested for relative potency following both single dose and booster dose vaccination assays. The study objective was to minimize the time factor exhausted in the evaluation processes of the inactivated fowl cholera vaccines. So it is planned to compare between single and booster dose vaccinations and their related potency. Correlation between protection associated with the single dose and booster dose vaccination were evaluated and average requirement for protection was $43.7 \%$ in single dose vaccination assay compared to $76.2 \%$ associated with booster dose vaccination assay. In the same concern, the correlation between both assays for the seroconversion was estimated using ELISA and the minimum requirement was $1.8 \times$ cut off value in the single dose vaccination assay compared to $2.25 \times$ cut off value in the booster dose vaccination assay. In conclusion, single dose vaccination assay could be valuable in the evaluation of inactivated fowl cholera vaccines through determination of protection indices and/or estimation of humoral immune response if the above mentioned data is considered.
\end{abstract}

Key words: Challenge and chicken, ELISA, Inactivated vaccine, Pasteurella multocida, Single dose

\section{INTRODUCTION}

Respiratory diseases are one of the major causes of economic losses to poultry industry. Fowl cholera is a septicemic respiratory complex caused by Pasteurella multocida, where it is highly common and widely distributed disease of poultry and other avian species (Rhoades et al., 1989; Xiao et al., 2015). P. multocida infections result in considerable losses to layer and breeder flocks in poultry industries worldwide. $P$. multocida lipopolysaccharide (LPS) is a primary stimulator of the host immune response and a critical determinant of bacterin protective efficacy (Harper et al., 2016). The disease can express itself in an acute or a chronic form. In the acute form, the clinical signs are seen only in the few hours before death as fever, ruffled feathers, mucus discharge from mouth, diarrhea and increased breathing rate. The chronic form of the disease can follow an acute stage or may be the only form of the disease present in the flock. Signs of this form generally linked to localized infection at wattles, sinuses, leg or wing joints, swollen eyes, twisted neck, rales and pin headed necrotic foci in the liver with a septicemic picture (Glisson et al., 2008; Akhtar et al., 2016).

Fowl cholera can be prevented by eliminating all reservoirs of infections and then preventing the re-entry of the organism into the property. Implementation of standard good management practices, effective sanitation regime and good biosecurity program will help prevention of fowl cholera (Blackall, 2003). Vaccination is considered as one of the common preventive measures worldwide to reduce the prevalence and incidence of disease (Kardos and Kiss, 2005). Pasteurella multocida exists in 16 different serovars and the most common serovars associated with fowl cholera outbreaks are serovars 1, 3 and 4. P. multocida vaccines based mainly on inactivated cells of P. multocida (Glisson et al., 2008). Evaluation and quality control of the efficacy of this vaccine are based mainly on vaccination challenge test by which the protective indices are estimated (OIE, 2017).

The immune system defends the organisms against infectious diseases and one of the major immunological 
defense mechanism is the humoral immune response, which is mediated by serum antibodies secreted by B cell (Weigend et al., 1997). Serological testing is a useful tool in explanation of immune status of the birds and the Enzyme Linked Immunosorbent Assay (ELISA) have been used to quantify antibodies against p. multocida, employing an array of antigens such as Outer Membrane Protein (Dogra et al., 2015)

The objective of the present work was to study the availability of using single dose vaccination assay in through determination of protection percent and humoral immune response in comparison to the booster dose vaccination assay of $P$. multocida inactivated vaccine in chickens.

\section{MATERIALS AND METHODS}

\section{Pasteurella multocida vaccines}

A total of 37 different inactivated $P$. multocida vaccinebatches yearling 2012 up to 2016, from different manufacturers sources either locally prepared or imported from abroad were tested by vaccination challenge assay method using virulent $P$. multocidain parallel to serological evaluation using ELISA.

\section{Pasteurella multocida strains}

Virulent Pasteurellamultocidaserovars 1, 3 and 4 were used to perform challenge test. These serovarswere supplied from the reference strain bank, CLEVB (Central Laboratory for the Evaluation of Veterinary Biologics).

\section{Laboratory animals}

\section{Chickens}

A total of 120 Hy-line Specific Pathogen Free (SPF) chickens aging 6-8 weeks were used for each fowl cholera vaccine batch tested to perform this study which starting from 2012 up to 2016. This birds were divided into three groups, the first one comprised 45 birds and received only one dose then challenged and serologically tested, the second group comprised also 45 birds and received both primary dose and three weeks later received a booster dose then challenged and serologically tested and finally the third group were 30 birds kept as negative unvaccinated group. All birds were vaccinated with the corresponding fowl cholera vaccine batch $(0.5 \mathrm{ml} /$ dose/chickens $)$ subcutaneously (OIE 2017).

\section{Swiss mice}

Six Swiss mice weighed about 20-25 gram, two for each $P$. multocida serovar were inoculated with the stock culture of $P$. multocida. This was done before every challenge test to rebuild the virulence of $P$. multocida serovars in a dose of 100500 CFU/ mouse intraperitoneally (OIE, 2017).

\section{Blood samples}

Twenty blood samples were collected from the jugular vein for each group per each tested batch of vaccines three weeks post vaccination in case of single dose vaccination assay or three weeks post the second dose of vaccination in case of booster dose vaccination assay, then sera were separated to be tested using ELISA.

\section{Challenge test}

The vaccinated birds were challenged with $2 \times 10^{2}$ to $3 \times 10^{2} \mathrm{CFU} /$ challenge dose from the different regained virulent $P$. multocida strains (15 vaccinated and five unvaccinated birds/each serovar) three weeks post vaccination in case of single dose vaccination assay or three weeks post the second dose of vaccination in case of booster dose vaccination assay. Mortalities were observed, recorded and re-isolation of the challenge strain were done from the internal organs (liver and heart blood) of dead cases and the Protective Indices (PI) were calculated using the following formula described by Timms and Marshall (1989).

$\mathrm{PI}=\%$ (M \& PML) controls $-\%$ (M \& PML) vaccinated $\times 100$

$$
\% \text { (M \& PML) controls }
$$

Where PI is the protective indices, $\mathrm{M}$ is the mortality and PML is the post-mortem lesions.

\section{ELISA}

ELISA was conducted on serum samples collected from all groups in different tested batches and the test performed according to standard procedures of the two different commercial kits used. The first one is $P$. multocida antibody test kit (Synbiotics Corporation, Cat. No.96-6527, USA) referred in this study as kit 1 while the second kit is $P$. multocida antibody test kit (IDEXX Laboratories. Inc., Cat. No. 99-09251, Netherland) which referred in this study as kit 2. ELISA was performed and interpreted as directed by the manufacturers.

\section{Statistical analysis}

Correlation coefficient which is the reciprocal relation between two or more variables was calculated and analyzed using SPSS program version 21 (2012). A statistic representing how closely two variables co-vary, it can vary from -1 (perfect negative correlation) through 0 (no correlation) to +1 (perfect positive correlation). 


\section{Ethical approval}

All the procedures have been carried out in accordance with the guidelines laid by institutional Ethics committee of central laboratory for evaluation of veterinary biologics (CLEVB) and in accordance with local lows and regulations.

\section{RESULTS}

Generally, fowl cholera vaccines are evaluated by sterility, safety and potency tests. Potency testing depends mainly on challenge test and determination of humoral immune response by ELISA as shown in table 1. A total of 32 out of 37 fowl cholera vaccine batches were tested and indicated satisfactory results for approval to be used in the poultry farms according to the Egyptian standards for evaluation of veterinary biologics (2004). According to the protection level obtained, the tested fowl cholera vaccine batches was grouped into seven categories. The protection percent in the first group comprises 6 batches out of 32 and was $41 \%$ in case of single dose vaccination assay compared with $70 \%$ in case of booster dose vaccination assay, in the second group comprises 3 batches out of 32 was $42 \%$ and $72 \%$, the third group comprises 11 batches (the highest average number of tested batches) out of 32 was $43 \%$ and $75 \%$, the fourth group comprises 2 batches out of 32 was $44 \%$ and $76 \%$, the fifth group comprises 7 batches out of 32 was $44 \%$ and $78 \%$, the sixth group comprises 2 batches out of 32 was $45 \%$ and $80 \%$ and the last seventh group comprises only one batch out of 32 was $47 \%$ and $82 \%$ in case of single and booster dose assays for each group respectively. On the other hand, table 1 also showed a comparison between the humeral immune response expressed ELISA mean titer for the same batch group at the same protection level. It was noticed that, the antibody titers at the protection level of $41 \%$ was parallel to 272 and 717 ELISA antibody titer for both types of ELISA kits respectively in case of single dose vaccination assay while it was 341 and 896 at the protection level of $70 \%$ in case of booster dose vaccination assay. Also it is clear that the antibody titer was increased as the protection level increased in a harmonious manner for both assays at all level of protections.

Table 1. Enzyme linked immuonosorbent assay mean titers and protection percent in Hy-line specific pathogen free chickens aging 6-8 weeks vaccinated with either single or booster dose vaccination assays of the satisfactory tested inactivated fowl cholera vaccines

\begin{tabular}{|c|c|c|c|c|c|c|c|c|c|c|c|c|}
\hline \multirow{3}{*}{$\begin{array}{l}\text { No. of tested } \\
\text { vaccines } \\
\text { batches }\end{array}$} & \multicolumn{6}{|c|}{ Single dose vaccination assay } & \multicolumn{6}{|c|}{ Booster dose vaccination assay } \\
\hline & \multicolumn{2}{|c|}{$\begin{array}{l}\text { ELISA Mean } \\
\text { Titer }\end{array}$} & \multicolumn{4}{|c|}{$\begin{array}{c}\text { Protection mean percent against } P \text {. } \\
\text { multocida serovar }\end{array}$} & \multicolumn{2}{|c|}{$\begin{array}{l}\text { ELISA Mean } \\
\text { Titer }\end{array}$} & \multicolumn{4}{|c|}{$\begin{array}{c}\text { Protection mean percent against } P . \\
\text { multocida serovar }\end{array}$} \\
\hline & Kit 1 & Kit 2 & 1 & 3 & 4 & Mean & Kit 1 & Kit 2 & 1 & 3 & 4 & Mean \\
\hline 6 & 272 & 717 & 40 & 43 & 40 & 41 & 341 & 896 & 70 & 71 & 70 & 70 \\
\hline 3 & 290 & 738 & 41 & 42 & 43 & 42 & 362 & 922 & 70 & 73 & 74 & 72 \\
\hline 11 & 298 & 766 & 42 & 42 & 45 & 43 & 373 & 958 & 77 & 73 & 75 & 75 \\
\hline 2 & 303 & 883 & 44 & 44 & 44 & 44 & 379 & 1104 & 78 & 74 & 74 & 76 \\
\hline 7 & 325 & 914 & 44 & 43 & 45 & 44 & 406 & 1143 & 78 & 81 & 79 & 78 \\
\hline 2 & 330 & 926 & 44 & 46 & 45 & 45 & 412 & 1157 & 80 & 81 & 80 & 80 \\
\hline 1 & 348 & 954 & 48 & 47 & 46 & 47 & 435 & 1192 & 85 & 83 & 82 & 83 \\
\hline Total 32 & 309 & 843 & & Mean & & 43.7 & 387 & 1053 & & Mean & & 76.2 \\
\hline
\end{tabular}

No: number, P: Pasturella, Kit 1: Pasturella Multocida ELISA KIT (Synbiotics, USA); Kit 2: Pasturella Multocida ELISA kit (IDEXX, Netherland)

By the same manner, table 2 illustrated that five fowl cholera vaccine batches out of 37 are evaluated as unsatisfactory, according to ESEVB (2004) where it got a protection level lower than $70 \%$ which is the minimum requirement for protection starting with $60 \%$ protection in two vaccine batches, $48 \%, 47 \%$ and $45 \%$ protection with one vaccine batch for each. Meanwhile, the protection achieved by the same batches when used only in single dose were $32 \%, 29 \%, 27 \%$ and $25 \%$ respectively.

As regards to the ELISA antibody titer of such unsatisfactory resulted batches, the corresponding antibody titers were 292 and 767 ELISA antibody titer with the protection rate $60 \%$ compared to 234 and 614 ELISA antibody titer with protection rate $32 \%$ in case of booster dose and single dose vaccination assays respectively. Also antibody titer decreased as the protection percent decreased in a parallel manner matched the immune status of the tested vaccine and birds in the rest unsatisfactory results of the tested batches.

Table 3, showed the average responses of birds vaccinated with either single or booster dose vaccination assays regarding both humoral responses and protection 
obtained. The average protection percent of all tested satisfactory batches was 76.2 with the booster dose compared to 43.7 with the single dose vaccination assay. Meanwhile, the average of measured ELISA antibody titer was 387 and 1053 compared to 309 and 843 for both kits and both assays respectively. On the other hand, the average protection percent of all tested unsatisfactory batches was 50 with the booster dose compared to 29 with the single dose vaccination assay. At the same time, the average of measured antibody titer was 242 and 619 compared to 194 and 495 for both kits and both assays respectively.

Table 2. Enzyme linked immuonosorbent assay mean titers and protection percent in Hy-line specific pathogen free chickens aging 6-8 weeks vaccinated with either single or booster dose vaccination assays of the unsatisfactory tested inactivated fowl cholera vaccines

\begin{tabular}{|c|c|c|c|c|c|c|c|c|c|c|c|c|}
\hline \multirow{3}{*}{$\begin{array}{l}\text { No. of } \\
\text { tested } \\
\text { vaccines } \\
\text { batches }\end{array}$} & \multicolumn{6}{|c|}{ Single dose vaccination assay } & \multicolumn{6}{|c|}{ Booster dose vaccination assay } \\
\hline & \multicolumn{2}{|c|}{$\begin{array}{l}\text { ELISA Mean } \\
\text { Titer }\end{array}$} & \multicolumn{4}{|c|}{$\begin{array}{c}\text { Protection mean percent against } P \text {. } \\
\text { multocida serovar }\end{array}$} & \multicolumn{2}{|c|}{$\begin{array}{l}\text { ELISA Mean } \\
\text { Titer }\end{array}$} & \multicolumn{4}{|c|}{$\begin{array}{c}\text { Protection mean percent against } P \text {. } \\
\text { multocida serovar }\end{array}$} \\
\hline & Kit 1 & Kit 2 & 1 & 3 & 4 & Mean & Kit 1 & Kit 2 & 1 & 3 & 4 & Mean \\
\hline 2 & 234 & 614 & 31 & 33 & 32 & 32 & 292 & 767 & 58 & 62 & 60 & 60 \\
\hline 1 & 193 & 497 & 30 & 28 & 29 & 29 & 241 & 621 & 50 & 44 & 50 & 48 \\
\hline 1 & 175 & 471 & 27 & 26 & 28 & 27 & 219 & 589 & 45 & 43 & 53 & 47 \\
\hline 1 & 174 & 399 & 25 & 24 & 26 & 25 & 217 & 499 & 44 & 43 & 48 & 45 \\
\hline Total 5 & 194 & 495 & & Mean & & 29 & 242 & 619 & & Mean & & 50 \\
\hline
\end{tabular}

No: number, P: Pasturella; Kit 1: Pasturella Multocida ELISA KIT (Synbiotics, USA); Kit 2: Pasturella Multocida ELISA kit (IDEXX, Netherland)

Table 3. Comparison and the correlation between mean of protection and ELISA titer afforded by single and booster fowl cholera vaccination in Hy-line specific pathogen free chickens aging 6-8 weeks

\begin{tabular}{|c|c|c|c|c|c|c|c|}
\hline \multirow{3}{*}{ Results } & \multirow{3}{*}{$\begin{array}{c}\text { No of } \\
\text { tested } \\
\text { batches }\end{array}$} & \multicolumn{3}{|c|}{ Single dose vaccination Assay } & \multicolumn{3}{|c|}{ Booster dose vaccination Assay } \\
\hline & & \multirow{2}{*}{$\begin{array}{l}\text { Protection } \\
\text { Mean }(\%)\end{array}$} & \multicolumn{2}{|c|}{ ELISA Mean Titer } & \multirow{2}{*}{$\begin{array}{l}\text { Protection } \\
\text { Mean (\%) }\end{array}$} & \multicolumn{2}{|c|}{ ELISA Mean Titer } \\
\hline & & & Kit 1 & Kit 2 & & Kit 1 & Kit 2 \\
\hline Satisfactory & 32 & 43.7 & 309 & 843 & 76.2 & 387 & 1053 \\
\hline Unsatisfactory & 5 & 29 & 194 & 495 & 50 & 242 & 619 \\
\hline
\end{tabular}

No: number; Kit 1: Pasturella Multocida ELISA KIT (Synbiotics, USA); Kit 2: Pasturella Multocida ELISA kit (IDEXX, Netherland)

\section{DISCUSSION}

Fowl cholera is a highly contagious and economically important disease of poultry worldwide. It is extremely important for poultry producers to be able to get a good vaccine against all poultry pathogens especially that they have great effect on this industry like fowl cholera. Evaluation of the efficacy of inactivated $P$. multocida or fowl cholera vaccine depends mainly on testing of its potency using vaccination- challenge test prior to sale and distribution (OIE, 2017).

Results of this study compared between two different vaccination assays either single dose or booster dose vaccination assays for the evaluation of inactivated fowl cholera vaccine using vaccination-challenge test and monitoring the immune response through determining the antibody titer against the inoculated vaccine using ELISA.

Depending on the minimum requirement of protection $(70 \%)$ which should be obtained after booster dose of vaccination of fowl cholera vaccine (Egyptian standards for evaluation of veterinary biologics, 2004), the satisfactory protection obtained in this study varies from $70 \%$ up to $83 \%$ giving rise a protection of $76.2 \%$ as an average in case of booster dose vaccination assay compared to $41 \%$ up to $47 \%$ giving rise a protection of 43.7 as an average in case of single dose vaccination assay. Meanwhile the average correlated ELISA antibody titer at this protection level was 387 and 1053 ELISA antibody titerin case of booster dose vaccination assay compared to 309 and 843 ELISA antibody titer obtained with single dose vaccination assay using ELISAkit 1 and 2 respectively. Analytical view of these antibody titer revealed that, it is equal to or more than the $2.25 x$ and $1.8 \times$ the calculated cut off value of both used kit 1 and kit 2. Also, these titers increased when the protection rate increased and decreased when the protection rate decreased as shown in tables 1 and 2. The same finding was obtained by Akhtar et al. (2016) found that the antibody titer in birds of group A injected with $1 \mathrm{ml}$ of vaccine and group B injected with $0.5 \mathrm{ml}$ of vaccine were 
4.513 and 4.07 respectively at primary vaccination, and 4.893 and 4.37 respectively after booster vaccination indicating significant increase in the antibody titre as compared to the titre of the primary vaccinaterd birds. The same criteriawere obtained by Jabbri and Moazeni (2005) who stated that fowl cholera vaccine consisted of serotypes 1,3 and $4 \mathrm{P}$. multocidastrains provided 70$100 \%$ protection against challenge with homologous strains. Also they found that the trivalent vaccine can induce immunogenic response in vaccinated chickens and so ELISA assay showed a considerable increase in antibody titer after twice vaccination of 6-8 weeks aged chicken. In the same concern, Belloc et al. (2008) assessed vaccine efficacy by measuring serum antibody titers 4 and 8 weeks after a single vaccination with several adjuvants included in $P$. multocida vaccines in chickens. The study concluded that the vaccine formulated with oil adjuvant MantonideISA70, ISA774 and W/O emulsion based on tween/span induced a strong immune response against $P$. multocida. AlsoJabbri and Moazeni (2005) reported that, the antibody measured with ELISA highly correlated with protection against challenge with virulent organisms. In the same concept, Pankaj and Arvind (2013) reported that a blocking ELISA was developed and standardized for the detection of antibodies to $P$. multocida in vaccinated animal. Meanwhile, Gong et al. (2016) detected serum antibody levels using indirect ELISA and reported that serum antibody levels of rPtfA subunit vaccine group and the attenuated live vaccine group were significantly higher than those of PBS group. In addition, there were no difference in antibody levels between the attenuated live vaccine group and $\mathrm{rPtfA}$ subunit vaccine group.

Regarding the average protection percent of all tested satisfactory batches, it was $76.2 \%$ with the booster dose compared to $43.7 \%$ with the single dose vaccination assay parallel to the average of measured antibody titer which was 378 and 1053 compared to 309 and 843 ELISA antibody titer for the both used kits and both assays respectively. On the other hand, the average protection percent of all tested unsatisfactory batches was 50 with the booster dose vaccination compared to $29 \%$ with the single dose vaccination assay parallel to the average antibody titer which was 242 and 619 compared to 194 and 495 ELISA antibody titerfor both kits and both assays respectively. Parvin et al. (2011) demonstrated the antibody titers following primary and booster vaccinations for four groups of different breeds of commercial birds and the study concluded that all groups of vaccinated birds induced a significant immune response after primary and booster vaccination without significant difference in antibody titers between different breeds. On the other hand, Perelman et al. (1990) recorded that ELISA test did not appear to be adequate for the evaluation of the degree of protection induced in turkey flocks where turkey poults vaccinated at one day old with inactivated $P$. multocida bacterin showed low titers of antibody as measured by ELISA but turkeys vaccinated at three and six weeks of age responded with a higher antibody titers and were resistant to virulent challenge with $P$. multocida. Akhtar et al. (2016) vaccinated birds with formalin killed fowl cholera bacterin, determined antibody titers using ELISA and challenged birds with virulent strain of P. multocida then concluded that the prepared formalin killed fowl cholera vaccine induce protective immune response and conferred protection against challenge protection infection caused by the virulent $P$. multocida strains.

By using a simple calculation regarding the finding of this study (Average protection with booster dose vaccination $(76.2 \%)$ and with single dose vaccination assay $(43.7 \%)$ and according to the minimum requirement in the ESEVB (2004) for veterinary vaccine evaluation which is $70 \%$ with the booster dose vaccination assay, the minimum requirement of protection associated with the single dose vaccination assay is $40.14 \%$.

It may be concluded that, the most important finding from the results of this study is the seroconversion of vaccinated birds with fowl cholera vaccine measured by ELISA concurrently with the protection obtained after challenge with the virulent $P$. multocida strains could be valuable and satisfactory in the evaluation of the efficacy of the fowl cholera vaccines using single dose vaccination assay and/or booster dose vaccination assay. Also, the minimum requirement of protection after challenge with the virulent $P$. multocida strains should be $40.14 \%$ or more in case of single dose vaccination assay.

\section{DECLARATIONS}

\section{Competing interest}

The authors have no conflict of interest.

\section{Consent to publish}

The authors declared the consent prior to publication of present article.

\section{Author's contributions}

Selim, Fatma, Fatma, Afaf and Abd Elhakim created the idea and the designed the study, collected the data, achieved all statistical analysis and wrote the paper. 


\section{REFERENCES}

Akhtar M, Rahman MDT, Ara MS, Rahman M, Nazir NH, Ahmed S, Hossen MDL and Rahman MDB (2016). Isolation of Pasteurella multocida from chickens, preparation of formalin killed fowl cholera vaccine, and determination of efficacy in experimental chickens. Journal of Advanced Veterinary and Animal Research, 3(1): 45-50. Doi: https://dx.doi.org/10.5455/javar. 2016. c130

Belloc C, Dupus L, Deville S, Aucouturier J and Laval A (2008). Evaluation of safety and immune response induced by several adjuvants included in Pasteurella multocida vaccines in chickens. Review Veterinary Medicine, 159 (7): $371-375$.

Blackall PJ (2003). Fowl cholera- an emerging disease in free range chickens. In queensland poultry science symposium. Gatton. Queensland.

Dogra V, Verma S, Singh G, Wani AH, Chahota R, Dhar P, Verma L and Sharma M (2015). Development of OMP based indirect ELISA to gauge the antibody titers in bovines against Pasteurella multocida. Iranian Journal of Veterinary Research, 16(4):350-356.

Egyptian standards for evaluation of veterinary biologics (2004). Central Laboratory for Evaluation of Veterinary Biologics, Ministry of Agriculture Land reclamation, Agriculture research center, pp. 161-164.

Glisson JR, Hofacre CL and Christensen JP (2008). Fowl cholera. In: Diseases of Poultry, Saif YM, Barnes HJ, Glisson JR, Fadly AM, McDougald LR and Swayne DE (Editors). Blackwell Publishing, Ames, Iowa, USA; pp. 739-758.

Gong Q, Qu N, Niu MF and Qin CL (2016). Evaluation of immunogenicity and protective efficacy of recombinant ptfA of avian Pasteurella multocida. Iranian Journal of Veterinary Recearch, 17(2): 84-88.

Harper M, John M, Edmunds M, Wright A, Ford M, Turni C, Blackall PJ, Cox A, Adler B and Boyce JD (2016). Protective efficacy afforded by live Pasteurella multocida vaccines in chickens is independent of lipopolysaccharide outer core structure. Vaccine, 34(14): 1696-1703. Doi: https://dx.doi.org/10.1016/j.vaccine.2016.02.017.

Jabbri AR and MoazeniJula GR (2005). Fowl cholera: Evaluation of trivalent Pasteurella multocida vaccine consisted of serotypes 1,3 and 4. Archives of Razi Institute, (59): 103- 111.

Kardos G and Kiss I (2005). Molecular Epidemiology Investigation of Outbreaks of Fowl Cholera in Geographically Related Poultry Flocks. Journal of Clinical Microbiology, 43(6): 2959-2961. Doi: https://dx.doi.org/10.1128/JCM.43.6.2959-2961.2005

Pankaj K and Arvind K (2013). Development and standardization of a blocking ELISA based on monoclonal antibody to $P$. multocida. Haryana Veterinarian, 52: 9092.

Parvin MS, Siddique MP and Islam MT (2011). Humoral immune response to fowl cholera vaccine in different breeds of commercial birds. Bangladesh Journal of Veterinary Medicine, 9 (2): 127 - 131. Doi: https://dx.doi.org/10.3329/bjvm. v9i2.13453

Perelman B, Hadash D, Meroz M, Gur- Lavie A, Abramson M and Samberg Y (1990). Vaccination of young turkeys against Fowl cholera. Avian pathology, 19: 131- 137. Doi: https://10.1080/03079459008418662

Rhoades KM, Rimler RB and Sandhu TS (1989). Pasteurellosis and Pseudo tuberculosis. Laboratory Manual for the Isolation and Identification of Avian Pathogens, H.G. Purchase, L. H. Arp, C.H. Domermuth, and J.E. Pearson (Eds). American Association of Avian Pathologists, Pennsylvania, pp. $14-21$.

Timms LM and Marshall N (1989). Laboratory assessment of protection given by experimental Pasteurella anatipestifer vaccine. British Veterinary Journal, 145: 483. Doi: https://dx.doi.org/10.1016/0007-1935(89)90059-6

Weigend S, Mielenz N and Lamont J (1997). Application of a nonlinear regression function to evaluate the kinetics of antibody response to vaccines in chicken lines divergently selected for multitrait immune response. poultry science, 76: 1248 - $1255 . \quad$ Doi: https://dx.doi.org/10.1093/ps/76.9.1248

World Organization of Animal Health (OIE) (2017). Fowl cholera, chapter 2.3.9. pp. 525- 530

Xiao K, Liu Q, Liu X, Hu Y, Zhao X and Kong Q (2015). Identification of the avian Pasteurella multocida phoP gene and evaluation of the effects of phoP deletion on virulence and immunogenicity. International Journal of Molecular Science, 17(12): 1-15. Doi: https://dx.doi.org/10.3390/ijms17010012. 